\title{
Skin optical clearing potential of disaccharides
}

\author{
Wei Feng \\ Rui Shi \\ Ning Ma \\ Daria K. Tuchina \\ Valery V. Tuchin \\ Dan Zhu
}




\title{
Skin optical clearing potential of disaccharides
}

\author{
Wei Feng, ${ }^{a, b, c, \dagger}$ Rui Shi, ${ }^{a, b, c, \dagger}$ Ning Ma, ${ }^{a, b, c}$ Daria K. Tuchina, ${ }^{d}$ Valery V. Tuchin, ${ }^{\text {d,e,f }}$ and Dan Zhu ${ }^{a, b, c, *}$ \\ ${ }^{a}$ Huazhong University of Science and Technology, Wuhan National Laboratory for Optoelectronics, Britton Chance Center for \\ Biomedical Photonics, 1037 Luoyu Road, Wuhan, Hubei 430074, China

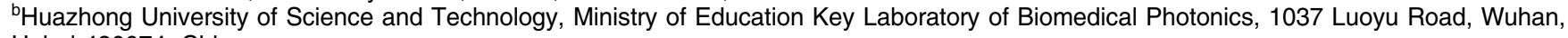 \\ Hubei 430074, China \\ 'Huazhong University of Science and Technology, Department of Biomedical Engineering, 1037 Luoyu Road, Wuhan, Hubei 430074, China \\ dSaratov State University, Research-Educational Institute of Optics and Biophotonics, 83 Astrakhanskaya Street, Saratov 410012, Russia \\ eInstitute of Precise Mechanics and Control RAS, 24 Rabochaya Street, Saratov 410028, Russia \\ 'Tomsk State University, Laboratory of Biophotonics, 36 Lenin's Avenue, Tomsk 634050, Russia
}

\begin{abstract}
Skin optical clearing can significantly enhance the ability of biomedical optical imaging. Some alcohols and sugars have been selected to be optical clearing agents (OCAs). In this work, we paid attention to the optical clearing potential of disaccharides. Sucrose and maltose were chosen as typical disaccharides to compare with fructose, an excellent monosaccharide-OCA, by using molecular dynamics simulation and an ex vivo experiment. The experimental results indicated that the optical clearing efficacy of skin increases linearly with the concentration for each OCA. Both the theoretical predication and experimental results revealed that the two disaccharides exerted a better optical clearing potential than fructose at the same concentration, and sucrose is optimal. Since maltose has an extremely low saturation concentration, the other two OCAs with saturation concentrations were treated topically on rat skin in vivo, and optical coherence tomography imaging was applied to monitor the optical clearing process. The results demonstrated that sucrose could cause a more significant increase in imaging depth and signal intensity than fructose. $\odot$ The Authors. Published by SPIE under a Creative Commons Attribution 3.0 Unported License. Distribution or reproduction of this work in whole or in part requires full attribution of the original publication, including its DOI. [DOI: 10.1117/1.JBO.21.8.081207]
\end{abstract}

Keywords: optical clearing; molecular dynamics simulation; optical clearing potential; rat skin in vivo; sucrose; fructose; optical coherence tomography imaging.

Paper 150883SSPRR received Jan. 4, 2016; accepted for publication Mar. 29, 2016; published online Apr. $25,2016$.

\section{Introduction}

Biomedical optical imaging techniques provide a powerful tool for accessing structural and functional information of tissues. ${ }^{1}$ However, the strong scattering of tissues induced by the mismatch in refractive indices (RIs) of different tissue components limits the penetration depth of light in tissue and reduces the contrast and depth of biomedical optical imaging. During the past years, tissue optical clearing (TOC) techniques were developed by introducing optical clearing agents (OCAs), ${ }^{2-4}$ which create promising opportunities for biomedical research because they could evidently reduce the scattering in tissue and extremely enhance imaging performance of various imaging techniques, including optical coherence tomography (OCT), 5,6 photoacoustic tomography, ${ }^{7,8}$ microscopic imaging, ${ }^{9-12}$ laser speckle contrast imaging, and so on. ${ }^{13-18}$

Skin is a protective layer of the body for preventing invasion of light or others into internal tissue, and is also a barrier layer for accessing physiological characteristics via noninvasive optical imaging. ${ }^{19}$ Undoubtedly, topical treatment of skin OCAs should be an available way for increasing the penetration of light into tissue and enhancing the optical imaging contrast, resolution, and depth. ${ }^{15,16,20}$ Therefore, skin optical clearing has attracted much attention. ${ }^{4,15,16,20-23}$ Various skin OCAs have been screened out based on experiments, including glycerol, dimethyl sulphoxide (DMSO), oleic acid, polyethylene glycol,

\footnotetext{
*Address all correspondence to: Dan Zhu, E-mail: dawnzh@mail.hust.edu.cn
}

tThese authors contributed equally to this work. butanediol, sorbitol, xylitol, glucose and fructose, and so on.,24-26 Khan et al. ${ }^{27}$ proposed an optical clearing potential (OCP), the ratio of reduced scattering coefficient before and after treatment of OCAs, to evaluate the optical clearing efficacy of skin. Some positive results have been obtained, e.g., hydroxyl-terminated organic compounds demonstrated higher OCP than others.

Thereafter, Hirshburg et al. further found that OCAs' OCP correlated with its ability for destabilizing the collagen structure because collagen located in the dermis is the main scattering source in skin. ${ }^{26,28,29}$ Then they employed molecular dynamics (MD) simulation to study the interaction between collagen and alcohols, and they predicted the OCP by means of analyzing the propensity to form hydrogen bonds and bridges. ${ }^{28,29}$ The MD theoretical simulation was further verified by the experiments, ${ }^{28,29}$ and the MD simulation would effectively avoid the blindness and time-consuming of experiments. Meanwhile, they found that increase of OCA molecular size and the number of hydroxyl groups could further increase the efficacy of TOC. ${ }^{28}$ Noting that sugars have hydroxyl groups, Wang et al. investigated sugars-induced skin optical clearing efficacy and found that fructose had the best OCP among monosaccharide-OCAs through MD simulation and experimental demonstration. ${ }^{16}$ Actually, disaccharides have a larger molecular size and more hydroxyl groups, but the OCP of disaccharides received little attention, and there is no comparative study between monosaccharides and disaccharides. Recently, some innovative TOC methods for ex vivo brain and other organs were developed, such as Scale ${ }^{30}$ ScaleS, ${ }^{11}$ SeeDB,${ }^{12}$ CUBIC, ${ }^{31}$ and so on; alcohol (glycerol and sorbitol) and sugar (fructose and sucrose) were also used, respectively. 
In addition, all of these methods usually include tedious steps or complex components, which make them unsuitable for in vivo skin because of their time-consumption and toxicity.

In this study, the OCP of sucrose and maltose, as the representatives of disaccharides, was investigated systematically by theoretical simulation, ex vivo and in vivo experiments, and compared with fructose. MD simulation was used to predict the OCP of the agents according to its propensity to form hydrogen bonds. Due to the three sugars having different saturation concentrations, various concentrations were prepared. After $e x$ vivo skin samples were immersed in each solution, the reduced scattering coefficients were measured by a commercially available spectrophotometer with an integrating sphere to evaluate the optical clearing efficacy. Further in vivo studies were performed by using OCT to evaluate the improvements in OCT imaging depth and signal intensity after topical treatment of saturated agents on in vivo rat skin.

\section{Materials and Methods}

\subsection{Chemical Agents}

Considering the solubility of disaccharides and theoretical investigation, sucrose and maltose were selected as the representatives to compare with fructose, which has been reported to have a greater OCP among the three monosaccharides. ${ }^{16}$ Figure 1 shows the chemical structures, respectively.

In this study, sucrose, maltose, and fructose were purchased from the Sinopharm Chemical Reagent Co., LTD (Shanghai, China). To evaluate the OPC of the OCAs, various agents with some concentration gradient were prepared for the ex vivo experiments. Their RIs were measured using an Abbe digital refractometer (WAY-2S, Shanghai YiCe Apparatus \&
Equipment Co., LTD, Shanghai, China). Since skin optical clearing methods have universality for various optical imaging techniques and the $\mathrm{pH}$ value of OCAs may produce influences on the fluorescence proteins or dyes, the $\mathrm{pH}$ values of each OCA with different concentrations were measured using $\mathrm{pH}$-Indicator strips (Sigma-Aldrich Co., Shanghai, China). The RIs and the $\mathrm{pH}$ values of each agent at different concentrations (Con.) were listed in Table 1.

\subsection{Molecular Dynamics Simulation}

MD simulation was employed to study the interactions between chemical agents and collagen. Here, the OCP of each agent was quantitatively evaluated by analyzing the number of hydrogen bonds formed between OCAs and collagen mimetic peptides. MD simulation software CHARMM (version-36, Chemistry at Harvard Macromolecular Mechanics, Harvard University, United States) with CHARMM22 force field and the carbohydrate force field was used. 1BKV (Protein Data Bank ID) and a regular GPO peptide $\left[(\mathrm{GPO})_{10}\right]_{3}(\mathrm{G}$, glycine; $\mathrm{P}$, proline; and $\mathrm{O}$, hydroxylproline) are representative collagen mimetic peptides for MD simulation. ${ }^{29}$ The information of the computational hardware is as follows: Intel(R) Core(TM) i5 CPU at 2.80 GHZ, 6 GB of physical RAM, Linux. The MD simulation of all agents proceeded in parallel, and the runtime was about $17 \mathrm{~h}$.

Each agent was applied to simulate with both $1 \mathrm{BKV}$ and GPO, respectively. On account of disaccharide molecular size limits, 10 disaccharide molecules (sucrose or maltose) were placed randomly around the collagen mimetic peptide at a radial distance of $12.0 \AA$ away from the cylindrical axis of the peptides. ${ }^{29}$ By contrast, 12 monosaccharide molecules (fructose) were placed in a similar way according to the previous

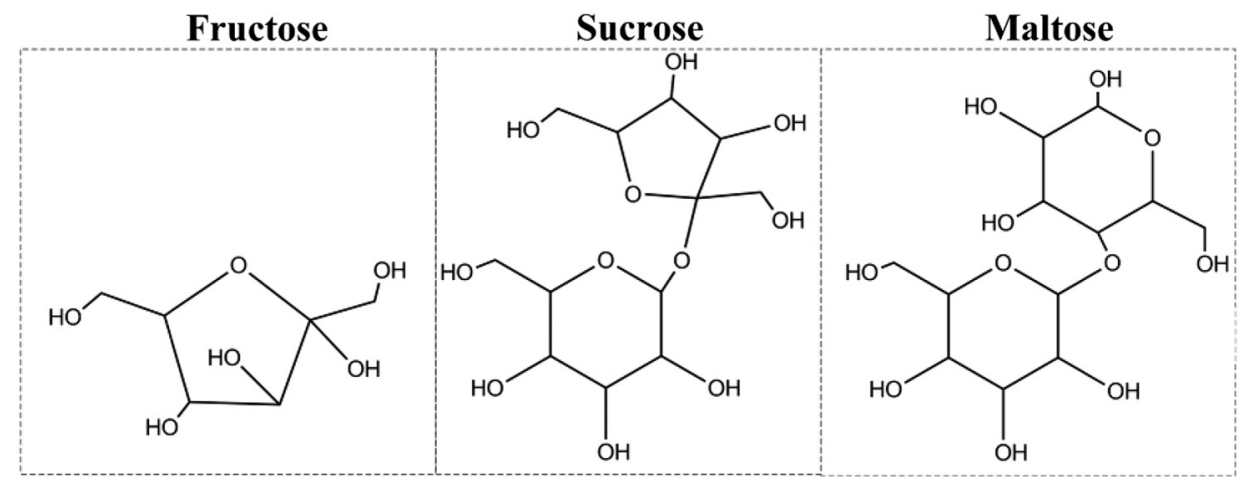

Fig. 1 Chemical structure of sucrose, maltose, and fructose.

Table 1 The Rls and the $\mathrm{pH}$ value of each agent at different Con. (M refers to mol/L).

\begin{tabular}{|c|c|c|c|c|c|c|c|c|c|c|c|c|}
\hline & Con. & $1 \mathrm{M}$ & $1.5 \mathrm{M}$ & $2 \mathrm{M}$ & $2.5 \mathrm{M}$ & $3 \mathrm{M}$ & $3.5 \mathrm{M}$ & $4 \mathrm{M}$ & $4.5 \mathrm{M}$ & $5 \mathrm{M}$ & $5.5 \mathrm{M}$ & $6 \mathrm{M}$ \\
\hline \multirow[t]{2}{*}{ Fructose } & RI & 1.3601 & 1.3744 & 1.3858 & 1.3996 & 1.4076 & 1.4265 & 1.4316 & 1.4475 & 1.4521 & 1.4589 & 1.4601 \\
\hline & $\mathrm{pH}$ & 6.5 & 6.5 & 6.5 & 6.5 & 6.5 & 6.0 & 6.0 & 6.0 & 6.0 & 5.5 & 5.5 \\
\hline \multirow[t]{2}{*}{ Sucrose } & RI & 1.3875 & 1.4111 & 1.4375 & 1.4611 & 1.4773 & - & - & - & - & - & - \\
\hline & $\mathrm{pH}$ & 6.5 & 6.5 & 6.5 & 7 & 7 & - & - & - & - & - & - \\
\hline \multirow[t]{2}{*}{ Maltose } & RI & 1.3830 & 1.4108 & 1.4471 & - & - & - & - & - & - & - & - \\
\hline & $\mathrm{pH}$ & 6 & 6 & 6 & - & - & - & - & - & - & - & - \\
\hline
\end{tabular}


study. ${ }^{16}$ Each production run lasted 600 picosecond (ps) and the coordinates were recorded every $1 \mathrm{ps}$. To prevent diffusion of the agent molecules away from the collagen, a cylindrical potential shell was applied, and the harmonic constraints were used to anchor the peptide. The detailed simulation process was reported by Hirshburg et al. ${ }^{29}$

The average numbers of hydrogen bonds between each agent and mimetic peptides during 600 ps were analyzed after the entire simulation. The cutoff radius of hydrogen bonds was set to $2.4 \AA .^{32}$ The number of hydrogen bonds formed between every molecule and collagen every 1 ps was counted to evaluate the skin OCP of sucrose, maltose, and fructose, respectively.

\subsection{Animal Preparation}

Male Sprague-Dawley rats (60 g, $n=40)$ were bought from Wuhan University Center for Animal Experiments (Wuhan, China). All animals were fed under a specific pathogen free level of feeding condition. The animal care and experimental procedures were approved by the Experimental Animal Management Ordinance of Hubei Province, China. Rats were anesthetized by an intraperitoneal injection of a mixture of $10 \%$ urethane and $2 \%$ - $\alpha$-chloralose with a dosage of $0.8 \mathrm{~mL} / 100 \mathrm{~g}$. After rats were thoroughly unhaired with depilatory cream, they were placed on the experimental platform.

\subsection{Ex Vivo Experiments}

Thereafter, we removed the dorsal skin and cleaned the subcutaneous fat, fascia, and blood completely. Then the fresh skin samples $(5 \mathrm{~cm} \times 5 \mathrm{~cm}, N=95)$ were immersed in phosphate buffer solution and kept under $4^{\circ} \mathrm{C}$ until experiments were performed in $<24 \mathrm{~h}$. The fresh dorsal skin samples were randomly divided into 19 groups according to Table 1.

A micrometer (Chengdu ChengLiang Tools Group Co., Ltd., Chengdu, China) was used to measure the thickness of skin sandwiched between two glass slides at four different directions. To reduce artificial measurement error, the measurements were done by the same person. The spectrophotometer (Lambda 950, PerkinElmer, Shelton, Connecticut) with an integrating sphere (150MM RSA ASSY, Labsphere, North Sutton), a dual-beam system, was applied to measure the reflectance and transmittance of the samples. The diameter of the integrating sphere is $150 \mathrm{~mm}$ with a circle reflectance-entrance $(r=12.70 \mathrm{~mm})$ and a transmittance-entrance. The transmittance-entrance combines two semicircles $(r=8.11 \mathrm{~mm})$ and one rectangle $(w \times h=16.22 \mathrm{~mm} \times 7.78 \mathrm{~mm}){ }^{33}$ The spectrophotometer's software WinLab was used to calibrate. Each measurement needed to scan a baseline, and $100 \%$ transmittance was performed without samples, $100 \%$ reflectance was performed by a reflectance standard (Labsphere), $0 \%$ transmittance and reflectance were in the absence of light to reflect the dark noises. After the skin samples were immersed into each solution for $45 \mathrm{~min}$, the thickness of the skin samples and the reflectance and transmittance spectra were measured again.

Based on the reflectance, transmittance, and thickness of each sample, the inverse adding-doubling method was applied to calculate the reduced scattering coefficient without introducing correction factors. Here, the skin RI and single scattering anisotropy were set as 1.4 and $0.8 .{ }^{34-36}$ Further, the reduced scattering ratio (RSR) was calculated as follows: ${ }^{28}$

$$
\mathrm{RSR}=\mu_{\mathrm{before}}^{\prime} / \mu_{\mathrm{after}}^{\prime}
$$

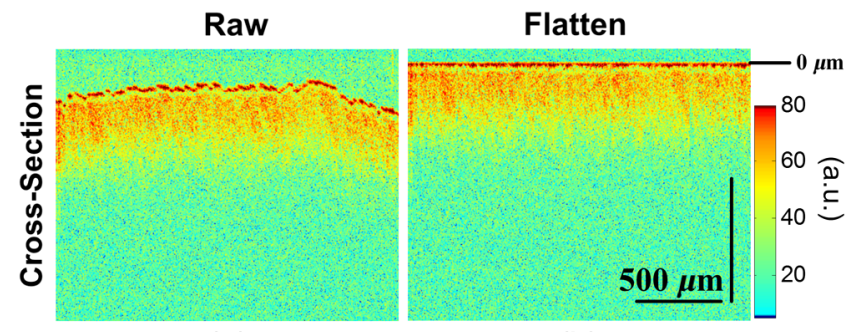

(a)

(b)

Fig. 2 (a) Original OCT image and (b) computer-flattened image. Bright color indicates the strong OCT signal intensity.

where $\mu_{\text {before }}^{\prime}$ and $\mu_{\text {after }}^{\prime}$ are the reduced scattering coefficient $(\lambda=635 \mathrm{~nm})$ before and after OCA treatment.

\subsection{Optical Coherence Tomography for Assessing Optical Clearing Potential In Vivo}

The imaging principle of OCT indicates that it has the capacity to image the tissue within 1 to $3 \mathrm{~mm}$ depth in vivo, ${ }^{37-39}$ which makes it a powerful tool to assess the optical clearing process in tissues. ${ }^{22,23,40}$ In this study, we used a commercial OCT system (OCP930SR, Thorlab Inc., Newton, New Jersey) working at $(930 \pm 5) \mathrm{nm}$ with $(100 \pm 5) \mathrm{nm}$ full-width-half-maximum, with an optical power of $2 \mathrm{~mW}$, a maximum image depth of $1.6 \mathrm{~mm}$, an axial resolution of $6.2 \mu \mathrm{m}$ (in air), and a lateral resolution about $10 \mu \mathrm{m}$. The saturated sucrose $(78.9 \%)$ or saturated fructose $(67.1 \%)$ induced skin optical clearing efficacy was assessed quantitatively by analyzing the improvements in imaging depth and signal intensity at the given depth.

Here, 12 rats were used for in vivo experiments. Initially, the prepared rats were placed on the experimental platform. The OCT B-scan images were recorded through the intact dorsal skin, tape stripped skin, and 6, 12, 18, 24, 30, and $36 \mathrm{~min}$ after topically applied saturated sucrose or saturated fructose on the skin, respectively. In order to reduce the mirror reflection caused by OCA, the OCA was quickly wiped away prior to acquiring the images and then was reapplied. It took about $30 \mathrm{~s}$ to wipe away and reapply the OCA, and $30 \mathrm{~s}$ acquire the images.

To facilitate analysis, the acquired images were flattened to enable averaging of the signal intensity profiles over the whole image so as to reduce the effects of the heterogenetic distribution of cutaneous absorbers and scatters. The flattening procedure was carried out with two steps: finding the $z$ position of the skin surface at each lateral $x$ position in the image and then translating the column of pixels at that $x$ to bring the skin surface to a common axial position. ${ }^{5}$ Figure 2 shows an example of flattening the skin by the image analysis. What is worth noting is that the flattening procedure may distort the tissue structure somewhat because it shifts pixels so that the skin surface is aligned at one constant $z$-axis position. However, such spatial distortion has minimal effect on the deduced light penetration since this study only paid attention the one-dimensional penetration of light into the skin. ${ }^{5}$

\section{Results}

\subsection{Hydrogen Bonds Formed Between Collagen and Agents}

Previous studies indicated that the higher-order structures of collagen could be stabilized by the hydration shell and 


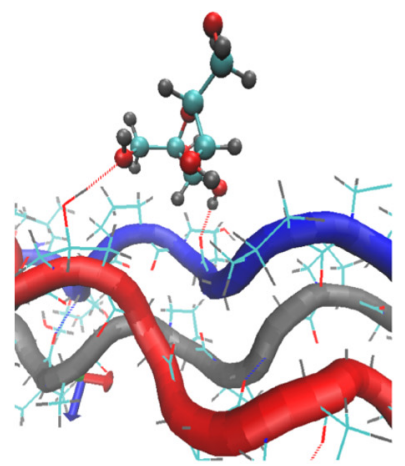

(a)

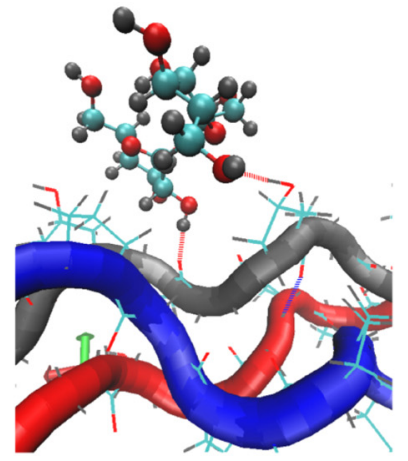

(b)

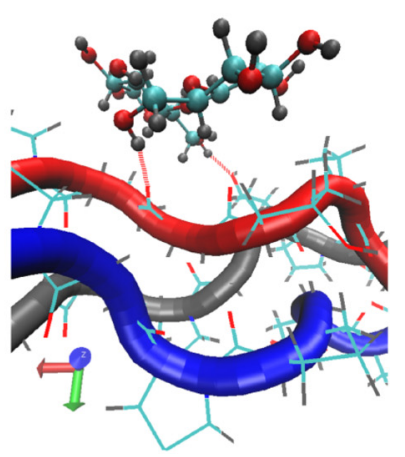

(c)

Fig. 3 Schematic representation of hydrogen bond formation of (a) fructose, (b) sucrose, and (c) maltose with representative collagen mimetic peptides, respectively.

water-mediated hydrogen bonds. ${ }^{28,32}$ Nevertheless, the formation of hydrogen bonds as well as hydrogen bond bridges between collagen and chemical agents could disrupt the hydration shell and water-mediated hydrogen bonds, ${ }^{29,32}$ which would destabilize the higher-order structure of collagen. Thus, the propensity of chemical agents to form hydrogen bonds could be used to predict the OCP of agents. Figure 3 shows the hydrogen bonds formed between the three agents and collagen mimetic peptide, it is expected that the more hydrogen bonds and hydrogen bond bridges an agent forms, the more effectively the hydration shell and water-mediated hydrogen bonds are disrupted.

The average numbers of hydrogen bonds formed between each agent and two collagen mimetic peptides per ps are calculated and listed in Table 2 . We find that sucrose forms the most

Table 2 Average number of hydrogen bonds formed.

\begin{tabular}{llll} 
& Fructose & Sucrose & Maltose \\
\hline Hydrogen bonds (\#/ps) & 1.254 & 1.307 & 1.292 \\
\hline
\end{tabular}

hydrogen bonds among the three agents. That is, sucrose has the strongest ability to disrupt the hydration shell and watermediated hydrogen bonds. Previous research has shown that the RSR increases as collagen solubility increases. ${ }^{41}$ Hence, it predicts that sucrose has the best skin OCP, while maltose is the second best and fructose is the worst, respectively, among the three agents.

\subsection{Ex Vivo Experiment for Reduced Scattering Ratio}

Further, to verify the prediction of MD simulation, the reduced scattering coefficient before and after skin samples were immersed into each agent was calculated, and then the RSR was deduced. The slope of the RSR was used to define the OCP of each agent. ${ }^{28}$ Figure 4 shows the RSR of skin samples treated with each agent at different concentrations for $45 \mathrm{~min}$, and it can be seen that the RSR increases with the concentration of an agent for the same OCA. Considering three concentration regions (A: 1-2 M; B: 1-3 M; C: 1-6 M) for each agent, different slopes were calculated separately. The results show that there is

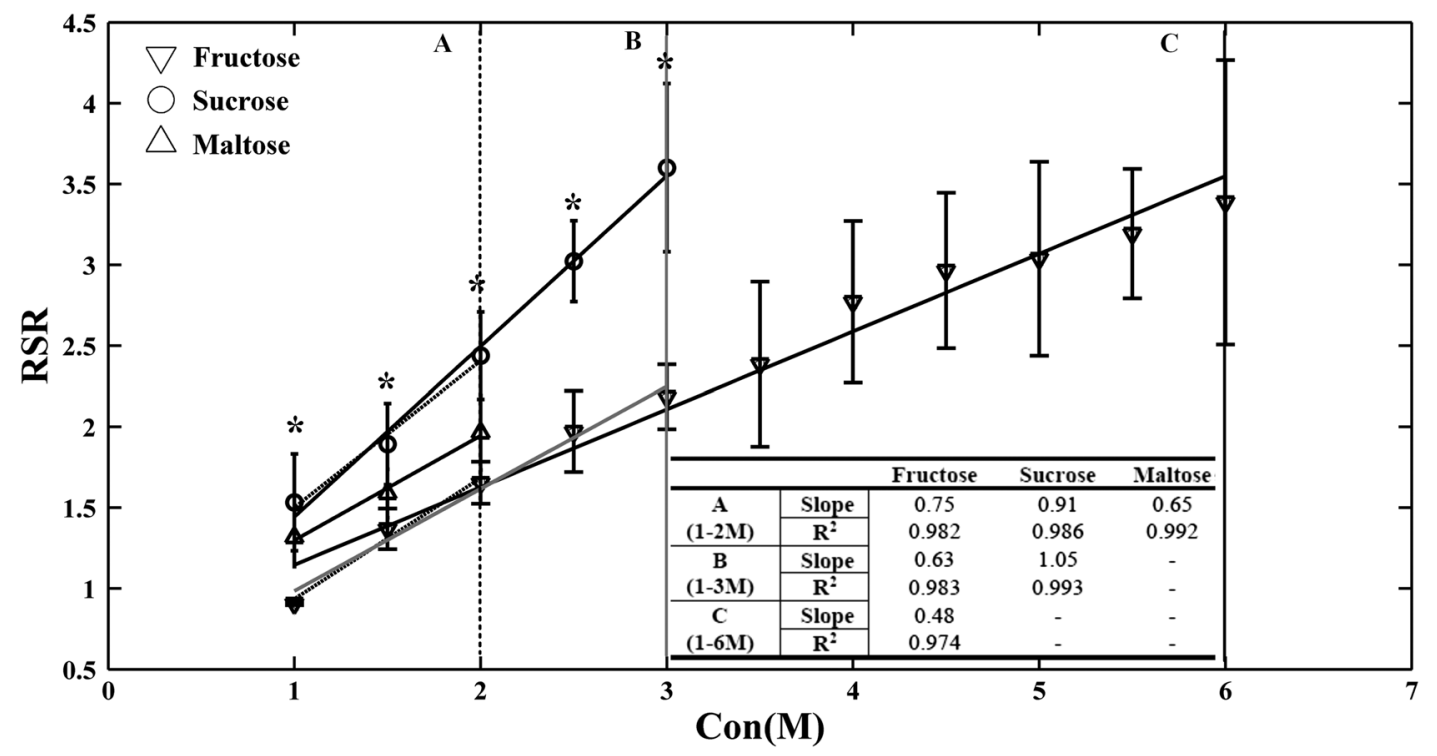

Fig. 4 RSR of ex vivo rat skin as a function of agent concentration. (a-c) The slope of each agent is obtained by linear regression analysis of RSR data in different saturated concentration regions (inset). The data are shown as mean \pm standard deviation $\left({ }^{*} p<0.05\right)$. 


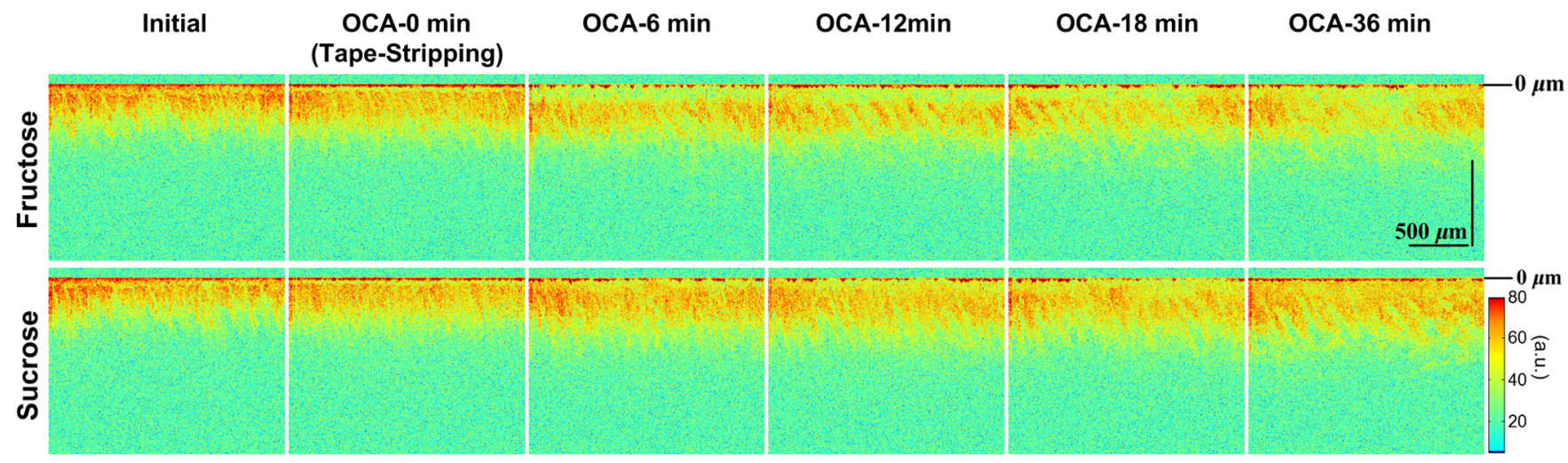

Fig. 5 Computer-flattened OCT images of rat dorsal skin treated with saturated fructose or saturated sucrose, respectively.

an excellent correlation $\left(R^{2}\right)$ between the experimental data and the fitted data for each agent. The slope in concentration region $\mathrm{A}$ is in a descending order of sucrose, fructose, and maltose. Likewise, in concentration region $\mathrm{B}$, the slope of sucrose is larger than that of fructose. According to Ref. 28, the slope is defined as the OCP of a chemical according to all the fitting data points, and the OCPs of sucrose, maltose, and fructose are $1.05,0.65$, and 0.48 , respectively. Thus, sucrose and fructose have the best and worst skin OCP among the three agents, which is consistent with the MD simulation results.

It should be noted that different chemicals have different saturation concentrations, and OCAs-induced optical clearing efficacy increases with its concentration. Furthermore, statistical analysis at common concentrations and the highest concentrations for fructose and sucrose were made, respectively. The results show that the RSR values of sucrose at the same concentration are obviously larger than those of other two agents $(p<0.05)$. However, there is no significant difference between the RSR of sucrose $(3 \mathrm{M})$ and fructose $(6 \mathrm{M})(p>0.05)$.

\subsection{Agent-Induced Improvements in OCT Imaging Depth and Signal Intensity}

Although MD simulation and ex vivo experimental results demonstrated that sucrose has a better OCP than that of fructose, the skin optical clearing efficacy caused by saturated sucrose is almost the same as that by saturated fructose. Moreover, both are on the basis of the direct interactions between the dermal collagen and the OCAs, which neglects the barrier of the stratum corneum that limits the penetration of OCAs into the dermis. In this work, the in vivo rat skin optical clearing performances of saturated fructose and saturated sucrose were evaluated using OCT.

Figure 5 shows the typical OCT results recorded through the intact rat skin in vivo, tape stripped skin, and 6, 12, 18, 36 min after the topical application of fructose (top row) or sucrose (bottom row) on the skin, respectively. By comparing the initial and the tape stripping, we find no significant changes, whereas after topical application of fructose or sucrose, the OCT imaging depth and signal intensity in the deeper region are enhanced gradually as optical clearing develops.

Figures 6(a)-6(b) show the typical normalized OCT signal intensity profiles after treatment of saturated fructose or sucrose for different times, respectively. It is found that OCAs' treatment can evidently increase the signal intensity. To quantitatively evaluate OCAs-induced changes in imaging depth, the depth at which the signal intensity attenuates to $1 / e$ of the normalized OCT intensity was calculated, respectively. The imaging depth can reach up to 450 or $550 \mu \mathrm{m}$ after treatment of saturated fructose or saturated sucrose for $36 \mathrm{~min}$, respectively, but the initial imaging depth is only $200 \mu \mathrm{m}$. Figure 6(c) demonstrates the changes in image depth versus treatment of OCA, and the slope of linear regression analysis was also used. The results show that the slope of saturated sucrose $(0.33)$ is higher than
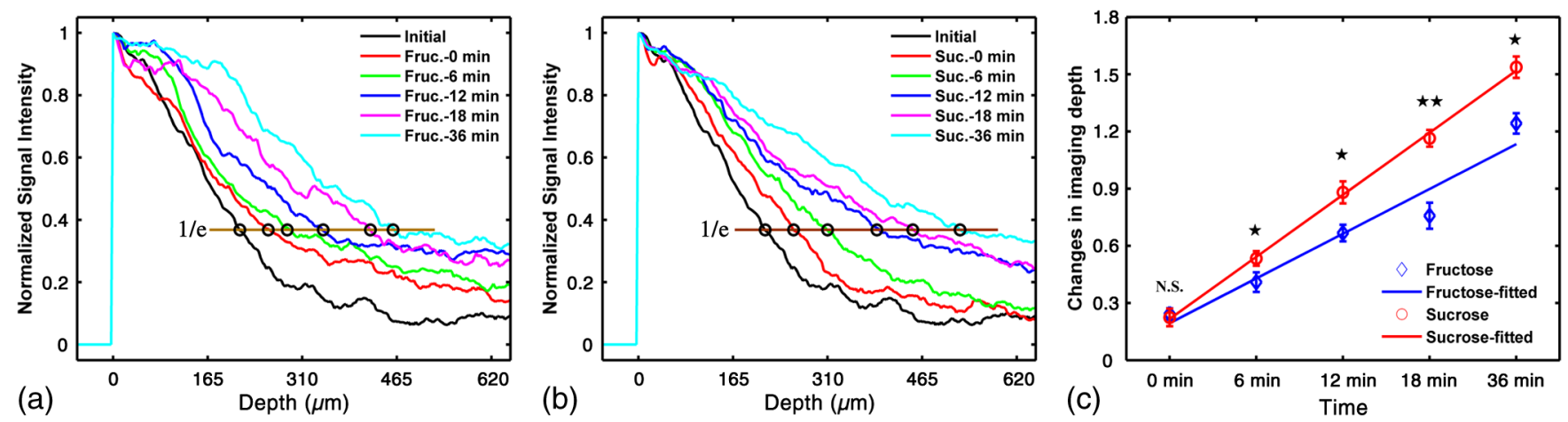

Fig. 6 Typical temporal dynamical profiles of normalized OCT signal intensity after treatment with (a) saturated fructose and (b) saturated sucrose, respectively. (c) The relative changes in imaging depth $\left(\Delta D / D_{0}\right)$ at different treatment time with the two saturated solutions. Data are mean \pm standard deviation, N.S. refers to no significant difference, ${ }^{*}$ means significant difference $(p<0.05)$, and ${ }^{* *}$ means extremely significant difference $(p<0.01)$. 

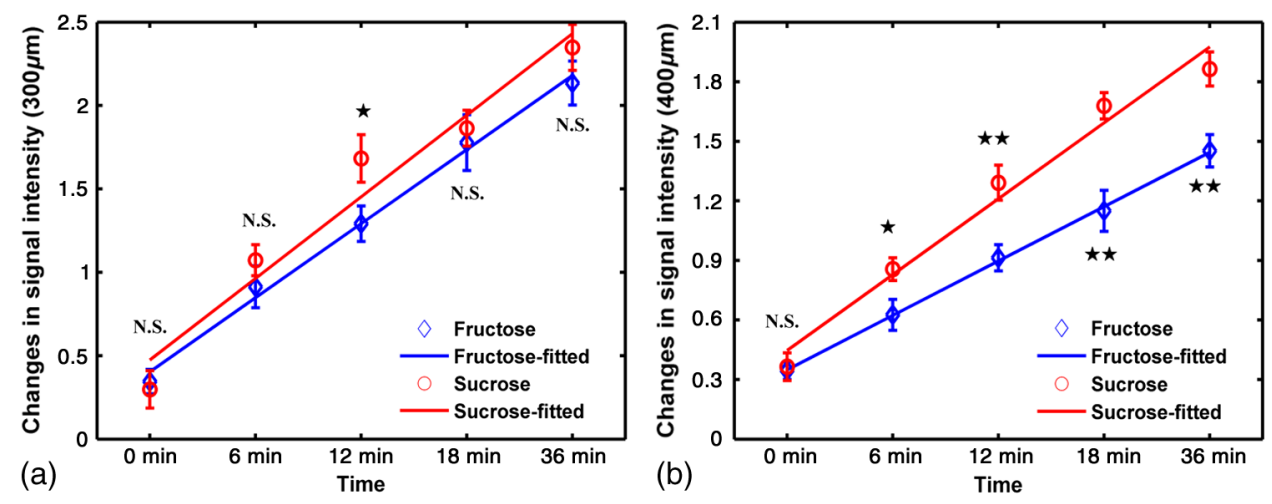

Fig. 7 Relative changes in signal intensity $\left(\Delta / / I_{0}\right)$ at the depth of (a) $300 \mu \mathrm{m}$ and (b) $400 \mu \mathrm{m}$ through the in vivo rat skin treated by saturated fructose and saturated sucrose, respectively. Data are mean \pm standard deviation, N.S. refers to no significant difference, * means significant difference $(p<0.05)$, and ${ }^{* *}$ means extremely significant difference $(p<0.01)$.

that of saturated fructose (0.23), respectively. Further statistical analysis using one-way analysis of variance (ANOVA) with SPSS (version 16.0, IBM) shows that there exists an extremely significant difference $(p<0.01)$ between the two slopes.

Furthermore, we calculated the changes in signal intensity at 300 and $400 \mu \mathrm{m}$ before and after OCA treatment for different times [see Figs. 7(a)-7(b)]. It demonstrates that both OCAs can significantly enhance the signal intensity at 300 and $400 \mu \mathrm{m}$. The linear regression slopes of sucrose and fructose are 0.49 and 0.44 for the changes in signal intensity at $300 \mu \mathrm{m}$, and 0.38 and 0.27 for that at $400 \mu \mathrm{m}$, respectively. Further statistical analysis using one-way ANOVA shows that there is an extremely significant difference for that at $400 \mu \mathrm{m}(p<0.01)$ between the linear regression slopes of fructose and sucrose, but no significant difference for that at $300 \mu \mathrm{m}(p>0.05)$.

\section{Discussion}

In this work, the optical clearing potential of disaccharides was investigated through the MD simulation, ex vivo, and in vivo experiments. Sucrose and maltose were chosen as typical disaccharides due to the limited availability of the force field files of disaccharides. Previous investigations showed that sugar-alcohols were common OCAs of skin optical clearing. For instance, fructose had better a OCP than glycerol, sorbitol, DMSO, and glycerol by the ex vivo experiments; ${ }^{26,41,42}$ and it was the best one among the three monosaccharides, such as glucose, fructose, and ribose from the MD simulation to experiments. ${ }^{16}$ Thus, we used fructose to compare with the disaccharides in this study.

The MD simulation revealed that the OCP is in the order of sucrose, maltose, and fructose. This is the reason that sucrose could form the most hydrogen bonds among the three agents, so molecular size and the numbers of hydroxyl groups play an important role in skin optical clearing. The occupation of the water-mediated hydrogen bonds sites by the OCAs-agent molecules would disrupt the hydration shell because of the formation of hydrogen bonds between OCAs-agents and collagen. ${ }^{26}$ Since disaccharides have a larger molecular size than monosaccharides, it has a bigger coverage on the collagen surface and observably shields water surrounding the collagen peptides. Thus, the OCP of disaccharides is stronger than that of monosaccharides, which implies that a higher OCP may be realized by extending the chain length for sugar-alcohols.
The theoretical prediction of the OCP of OCAs was demonstrated by ex vivo experiments. The results indicate that sucrose has a larger OCP than fructose, which is inconsistent with that reported in previous work. ${ }^{41}$ The correlations $\left(R^{2}\right)$ between the experimental data and the fitted data in Ref. 41 were only 0.73 and 0.78 , for sucrose and fructose, respectively, whereas the $R^{2}$ reach up to 0.993 and 0.974 in this work.

Undoubtedly, the OCP is an important parameter to evaluate the OCA-induced skin optical clearing efficacy. However, each chemical has a different solubility or saturation concentration. It is well known that a high concentration of OCA can induce better optical clearing efficacy for the same chemical. Therefore, OCA-induced optimal optical clearing efficacy not only depends on the OCP, but also on its saturation concentration. Thus, it is easy to understand why fructose with a lower OCP and higher concentration (6 M) could cause almost the same ex vivo skin optical clearing efficacy as sucrose with a higher OCP and lower concentration $(3 \mathrm{M})$.

In addition, both the theoretical prediction and ex vivo experiments neglect the barrier function of the epidermis. Therefore, OCAs-induced in vivo skin optical clearing efficacy may be relative to the penetrability of OCAs. Since the saturation concentration of maltose is extremely low, for the in vivo skin optical clearing experiments, only the saturated sucrose was compared with the saturated fructose. Results indicated that both OCAs would increase the signal intensity in the deeper region. Fructose treatment decreases the signal intensity in the shallower region, whereas this situation was less obvious for sucrose treatment. This inconsistent phenomenon should be the result of the different influences of the OCAs on surface reflectivity, which was also observed by other investigators when they used different OCAs. For instance, Ghosn et al., ${ }^{43}$ and Wen et al. ${ }^{5}$ reported similar changes with our results of the fructose treatment; and Zhong et al. reported that optical clearing of human skin enhanced the OCT signal at all depths; ${ }^{44} \mathrm{Xu}$ et al. found that optical clearing of porcine skin in vitro increased the reflectance from deeper dermis although the OCT signal from superficial skin did not change. ${ }^{45}$ Meanwhile, the deeper penetration depth promoted the enhancement of image contrast, which was demonstrated by Wen et al., ${ }^{5}$ Larin et al., ${ }^{6}$ Proskurin et al., ${ }^{46}$ and Bonesi et al. ${ }^{47,48}$

OCT imaging results showed 1.5 to 2.5 times enhancements in penetration depth or signal intensity after topical application of saturated fructose and sucrose, respectively. The imaging 
depth for the intact rat skin was only about $200 \mu \mathrm{m}$, which was insufficient for obtaining the dermal information, whereas after OCAs treatment, the imaging depth could reach 450 to $550 \mu \mathrm{m}$, which can be almost enough to penetrate the whole dermis. That makes it possible to access blood flow distribution information, track tumor cell development, and monitor interventional treatment of vascular diseases and so on, combined with various optical imaging modalities, ${ }^{1,49}$ e.g., photoacoustic tomography, ${ }^{7,8}$ fluorescence imaging, ${ }^{9-12}$ and laser speckle contrast imaging. ${ }^{13-18}$ Especially, the $\mathrm{pH}$ of fructose decreases with its concentration, which shows a meta-acid with a $\mathrm{pH}$ of 5.5 at a concentration of $6 \mathrm{M}$, whereas the $\mathrm{pH}$ of the sucrose increases with its concentration, which is neutral with a $\mathrm{pH}$ of 7 at a concentration $3 \mathrm{M}$. The acid materials may quench fluorescent protein, ${ }^{50}$ which indicates that sucrose will be more befitting for fluorescence imaging.

In addition, in vivo skin optical clearing efficacy also depends on the penetration of OCAs. If some chemical penetration enhancers, such as zone, thiazone, DMSO and so on, are introduced, ${ }^{13,42,51}$ the imaging depth will be further enhanced. This information is expected to play a much more significant role in clinical application ${ }^{21}$ and basic research.

\section{Conclusion}

In this work, the OCP of sucrose, maltose, and fructose was predicted by MD simulation, which was verified by the ex vivo experiments. The results indicated that sucrose had the best skin OCP, but there is no significant difference between sucrose at $3 \mathrm{M}$ and fructose at $6 \mathrm{M}$ for induced skin optical clearing efficacy. The in vivo OCT imaging experiment indicated that the imaging depth and signal intensity were obviously improved after topical treatment with the saturated fructose and sucrose, and that sucrose is better than fructose. This study combined MD simulation with ex vivo and in vivo experiments to hunt for a novel preferable skin OCA from common disaccharides, which expands the reagent library of skin OCAs. It is hoped that sucrose will play an important role in optical clearing-based application and research.

\section{Acknowledgments}

This study was supported by the National Nature Science Foundation of China (Grants Nos. 812111313, 81171376, 91232710, and 31571002), the Science Fund for Creative Research Group of China (Grant No. 61421064), the seed project of Wuhan National Laboratory for Optoelectronics, and the Russian Science Foundation (Grant No. 14-15-00186).

\section{References}

1. S. M. Daly and M. J. Leahy, “'Go with the flow': a review of methods and advancements in blood flow imaging," J. Biophotonics 6(3), 217255 (2013)

2. D. Zhu et al., "Recent progress in tissue optical clearing," Laser Photonics Rev. 7(5), 732-757 (2013)

3. E. A. Genina et al., "Tissue optical immersion clearing," Expert Rev. Med. Devices 7(6), 825-842 (2010).

4. E. A. Genina et al., "Optical clearing of biological tissues: prospects of application in medical diagnostics and phototherapy," J. Biomed. Photonics Eng. 1(1), 22-58 (2015).

5. X. Wen et al., "Enhanced optical clearing of skin in vivo and optical coherence tomography in-depth imaging," J. Biomed. Opt. 17(6), 066022 (2012).
6. K. V. Larin et al., "Optical clearing for OCT image enhancement and in-depth monitoring of molecular diffusion," IEEE J. Sel. Top. Quant. 18(3), 1244-1259 (2012).

7. Y. Zhou, J. Yao, and L. H. V. Wang, "Optical clearing aided photoacoustic microscopy with enhanced resolution and imaging depth," Opt. Lett. 38(14), 2592-2595 (2013).

8. Y. Liu et al., "Optical clearing agents improve photoacoustic imaging in the optical diffusive regime," Opt. Lett. 38(20), 4236-4239 (2013).

9. K. Tainaka et al., "Whole-body imaging with single-cell resolution by tissue decolorization," Cell 159(4), 911-924 (2014).

10. B. Hou et al., "Scalable and DiI-compatible optical clearance of the mammalian brain," Front. Neuroanat. 9, 19 (2015).

11. H. Hama et al., "ScaleS: an optical clearing palette for biological imaging," Nat. Neurosci. 18(10), 1518-1529 (2015).

12. M. T. Ke et al., "SeeDB: a simple and morphology-preserving optical clearing agent for neuronal circuit reconstruction," Nat. Neurosci. 16(8), 1154-1161 (2013).

13. D. Zhu et al., "Imaging dermal blood flow through the intact rat skin with an optical clearing method," J. Biomed. Opt. 15(2), 026008 (2010).

14. J. Wang et al., "Switchable skin window induced by optical clearing method for dermal blood flow imaging," J. Biomed. Opt. 18(6), 061209 (2013).

15. R. Shi et al., "Accessing to arteriovenous blood flow dynamics response using combined laser speckle contrast imaging and skin optical clearing," Biomed. Opt. Express 6(6), 1977-1989 (2015).

16. J. Wang et al., "Sugars Induced skin optical clearing from molecular dynamics simulation to experimental demonstration," IEEE J. Sel. Top. Quantum Electron. 20(2), 7101107 (2014).

17. J. Wang et al., "Review: tissue optical clearing window for blood flow monitoring," IEEE J. Sel. Top. Quantum Electron. 20(2), 6801112 (2014).

18. J. Wang et al., "An innovative transparent cranial window based on skull optical clearing," Laser Phys. Lett. 9(6), 469-473 (2012).

19. G. Mahe et al., "Assessment of skin microvascular function and dysfunction with laser speckle contrast imaging," Circ-Cardiovasc. Imaging 5(1), 155-163 (2012).

20. J. Wang et al., "Ear skin optical clearing for improving blood flow imaging," Photonics Lasers Med. 2(1), 37-44 (2013).

21. C. Liu et al., "Quantitative evaluation of enhanced laser tattoo removal by skin optical clearing," J. Innov. Opt. Health Sci. 8(03), 1541007 (2015).

22. E. A. Genina et al., "Optical coherence tomography monitoring of enhanced skin optical clearing in rats in vivo," J. Biomed. Opt. 19(2), 021109 (2014).

23. Z. Deng et al., "Viscous optical clearing agent for in vivo optical imaging," J. Biomed. Opt. 19(7), 076019 (2014).

24. Z. Mao et al., "Influence of alcohols on the optical clearing effect of skin in vitro," J. Biomed. Opt. 13(2), 021104 (2008).

25. N. Kuznetsova et al., "Sugars and polyols inhibit fibrillogenesis of type I collagen by disrupting hydrogen-bonded water bridges between the helices," Biochemistry 37(34), 11888-11895 (1998).

26. J. M. Hirshburg et al., "Collagen solubility correlates with skin optical clearing,” J. Biomed. Opt. 11(4), 040501 (2006).

27. M. H. Khan et al., "Optical clearing of in vivo human skin: implications for light-based diagnostic imaging and therapeutics," Lasers Surg. Med. 34(2), 83-85 (2004).

28. J. M. Hirshburg, Chemical agent induced reduction of skin light scattering, PhD Dissertation, Texas A\&M University (2009).

29. J. M. Hirshburg et al., "Molecular basis for optical clearing of collagenous tissues," J. Biomed. Opt. 15(5), 055002 (2010).

30. H. Hama et al., "Scale: a chemical approach for fluorescence imaging and reconstruction of transparent mouse brain," Nat. Neurosci. 14(11), 1481-1488 (2011).

31. E. A. Susaki et al., "Whole-brain imaging with single-cell resolution using chemical cocktails and computational analysis," Cell 157(3), 726-739 (2014).

32. K. M. Ravikumar and W. Hwang, "Region-specific role of water in collagen unwinding and assembly," Proteins 72(4), 1320-1332 (2008).

33. X. Zhong et al., "Effect of light beam on measurements of reflectance and transmittance of turbid media with integrating sphere: Monte Carlo simulation," Front. Optoelectron. 8(2), 203-211 (2015). 
34. A. N. Bashkatov et al., "Monte Carlo study of skin optical clearing to enhance light penetration in the tissue: implications for photodynamic therapy of acne vulgaris," Proc. SPIE 7022, 702209 (2007).

35. E. A. Genina et al., "Possibility of increasing the efficiency of laserinduced tattoo removal by optical skin clearing," Quantum Electron. 38(6), 580-587 (2008).

36. I. V. Meglinski et al., "Study of the possibility of increasing the probing depth by the method of reflection confocal microscopy upon immersion clearing of near-surface human skin layers," Quantum Electron. 32(10), 875-882 (2002).

37. Y. Wu et al., "Noninvasive optical coherence tomography monitoring of structure and hydration changes of human corneas in different preservation media," J. Biomed. Opt. 16(2), 026015 (2011).

38. S. Yousefi et al., "Assessment of microcirculation dynamics during cutaneous wound healing phases in vivo using optical microangiography," J. Biomed. Opt. 19(7), 076015 (2014).

39. C. Liu et al., "Improvement of tissue analysis and classification using optical coherence tomography combined with Raman spectroscopy," J. Innov. Opt. Health Sci. 8(4), 1550006 (2015).

40. A. F. Pena et al., "Monitoring of interaction of low-frequency electric field with biological tissues upon optical clearing with optical coherence tomography," J. Biomed. Opt. 19(8), 086002 (2014).

41. J. M. Hirshburg et al., "Correlation between collagen solubility and skin optical clearing using sugars," Lasers Surg. Med. 39(2), 140-144 (2007).

42. X. Xu and R. K. Wang, "Synergistic effect of hyperosmotic agents of dimethyl sulfoxide and glycerol on optical clearing of gastric tissue studied with near infrared spectroscopy," Phys. Med. Biol. 49(3), 457-468 (2004).
43. M. G. Ghosn et al., "Monitoring of glucose permeability in monkey skin in vivo using optical coherence tomography," J. Biophotonics 3(1-2), 25-33 (2010).

44. H. Zhong et al., "Synergistic effect of ultrasound and thiazone-PEG 400 on human skin optical clearing in vivo," Photochem. Photobiol. 86(3), 732-737 (2010)

45. X. Xu, Q. Zhu, and C. Sun, "Assessment of the effects of ultrasoundmediated alcohols on skin optical clearing," J. Biomed. Opt. 14(3), 034042 (2009).

46. S. G. Proskurin and I. V. Meglinski, "Optical coherence tomography imaging depth enhancement by superficial skin optical clearing," Lasers Phys. Lett. 4(11), 824-826 (2007).

47. M. Bonesi, S. G. Proskurin, and I. V. Meglinski, "Imaging of subcutaneous blood vessels and flow velocity profiles by optical coherence tomography," Laser Phys. 20(4), 891-899 (2010).

48. M. Bonesi, S. Matcher, and I. Meglinski, "Doppler optical coherence tomography in cardiovascular applications," Laser Phys. 20(6), 1491-1499 (2010).

49. M. J. Leahy et al., "Biophotonic methods in microcirculation imaging," Med. Laser Appl. 22(2), 105-126 (2007).

50. T. Yu et al., "A rapid and PI-compatible optical clearing method for brain tissue based on sugar/sugar-alcohol," J. Biomed. Opt. 21(8), 081203 (2016).

51. A. C. Williams and B. W. Barry, "Penetration enhancers," Adv. Drug Deliver. Rev. 56(5), 603-618 (2004).

Biographies for the authors are not available. 